\title{
Anesthesia risks associated with pediatric imaging
}

\author{
Charles Cauldwell
}

Received: 14 May 2011 / Accepted: 17 May 2011 /Published online: 18 June 2011

(C) Springer-Verlag 2011

\begin{abstract}
Any medical procedure, whether diagnostic or therapeutic, should produce at least as much benefit as risk. This principle applies from the simplest, seemingly innocuous test to the most complicated invasive operation. Knowledge of the benefits and risks, and analysis of the risk-to-benefit ratio, therefore, should precede the decision to go forward with any procedure. Radiological imaging is not immune to this situation.
\end{abstract}

\section{Risks of imaging}

The commentary by Greenberg [1] in this issue of Pediatric Radiology attempts to compare some of the risks associated with CT and MRI. As CT technology has advanced and multi-slice scanners have reduced the scan time significantly, it has become feasible to image children without the need for anesthesia or sedation. However, CT involves exposure to ionizing radiation and has been linked with an increased risk of the development of cancer that is inversely proportional to the age at time of exposure, because children are more radiosensitive and they have a longer time to develop cancer. One obvious response would be the replace the use of CT with imaging techniques that don't involve ionizing radiation. One such technique is MRI. However, MRI imaging takes considerably longer and is sensitive to movement, so some, but not all, children need anesthesia to successfully complete an MRI scan. Greenberg is appropriate to point out the increase in overall risk to the patient when he or she needs general anesthesia for MRI.

\section{Cauldwell ( $\square)$}

Department of Anesthesia and Perioperative Care,

University of California San Francisco,

521 Parnassus Ave., CS 450-A,

San Francisco, CA 94143-0648, USA

e-mail: cauldwec@anesthesia.ucsf.edu

\section{Short-term risks of anesthesia}

General anesthesia is relatively safe for children. A recent retrospective study from Melbourne Children's Hospital revealed ten deaths related to 101,885 anesthetics, a risk of 1 in 10,188 [2]. All 10 deaths involved children who had pre-existing medical conditions that were identified as factors in their outcome, leading to the conclusion that anesthesia on relatively healthy children is quite safe. Delivering an anesthetic outside the operating room presents some challenges. The physical environment is typically not designed for delivery of anesthesia, e.g., arrangement of the gas/vacuum connections and electrical outlets, and relation of scanning space and location of the anesthesia machine. The induction of anesthesia may need to occur outside the scanning area and require the movement of an anesthetized patient. Different hospitals have approached this problem in different ways. One model is to anesthetize (or deeply sedate) the patient in an ICU or treatment room then transport the patient to the imaging area. (From the standpoint of overall risk, deep sedation, as defined by guidelines of the American Academy of Pediatrics and the American Society of Anesthesiologists, does not differ significantly from general anesthesia. Preprocedure evaluation, NPO parameters and monitoring should not be substantially different between the two levels of sedation/anesthesia and anyone performing deep sedation should be able to rescue the patient from general anesthesia according to the above guidelines.) Another model is to provide a space immediately adjacent to the imaging area where induction can occur. Anesthesia administered near an MRI scanner presents issues related to the magnetic field. In either model, moving an anesthetized patient from one place to another is logistically challenging and increases the risk. The study Greenberg 
presented attempted to assess this risk and showed 3 deaths in 11,700 anesthetics, a risk of 1 in 3,900 [3]. But the small numerator in this study makes the real risk hard to evaluate and all three patients had co-morbidities, similar to the Melbourne study.

Other factors that would increase the risk of anesthesia for MRI would be inadequate NPO time or a request for oral contrast agent immediately prior to the scan, or the need for breath-holds for certain sequences, which might lead a decision to intubate the trachea for control of ventilation rather than maintaining spontaneous ventilation via nasal cannula, which might otherwise be done.

\section{Long-term risks of anesthesia}

During the last few years, it has become apparent that there are neurodevelopmental risks of general anesthesia, particular for children younger than 2 or 3 years of age. Multiple experiments in animals, primarily rats, but also primates, have shown that during the period of synaptogenesis, exposure to anesthetic agents leads to apoptotic neurodegeneration and long-term neurocognitive impairment [46]. The Anesthetic and Life Support Drugs Committee of the FDA met in 2007 to consider anesthetic neurotoxicity in children. The final outcome was that there were no clinical data to demonstrate neurotoxicity but that future research was necessary; no changes in anesthetic practice were indicated at that time [7]. The FDA continues to be involved and a second advisory committee met in March. There are ongoing clinical trials investigating the safety of anesthetic agents but they will take several years to achieve consensus [8]. A study just published from Denmark demonstrated that academic performance in the ninth grade did not differ between a group who underwent hernia repair prior to 1 year of age and controls (Hansen et al. [9]). Meanwhile, some agents and maneuvers have been investigated that might provide neuroprotection. Lithium, hypothermia and xenon have been investigated in animal models $[4,10]$.

\section{Conclusion}

How can the radiologist minimize the risk to a child who needs imaging to define disease? MRI can't substitute for all CT scans. The first question to answer: what is the best imaging procedure for the clinical situation? That requires communication between the pediatric team and the pediatric radiologist. US might provide enough information to answer the question, without the use of either radiation or anesthesia, although it is more operator-dependent. It might be that no study is necessary at the time. A recent study in Pediatrics showed that for children with blunt head trauma, observation prior to CT scanning led to a lower use of CT scans with no difference in outcome [11]. Another approach is to minimize the exposure to radiation during CT. The Image Gently campaign is a program to reduce radiation exposure through education of radiologists and technologists. But when all is said and done, a CT scan might be the best way to answer the question posed to the radiologist. There is no free lunch.

\section{References}

1. Greenberg SB (2011) Rebalancing the risks of CT and MR imaging. Pediatr Radiol 41. doi:10.1007/s00247-011-2159-3

2. Van der Griend BF, Lister NA, McKenzie IM et al (2011) Postoperative mortality in children after 101,885 anesthetics at a tertiary pediatric hospital. Anesth Analg [Epub ahead of print] May 5

3. Girshin M, Shapiro V, Rhee A et al (2009) Increased risk of general anesthesia for high-risk patients undergoing magnetic resonance imaging. J Comput Assist Tomogr 33:312-315

4. Creeley CE, Olney JW (2010) The young: neuroapoptosis induced by anesthetics and what to do about it. Anesth Analg 110:442-448

5. Mellon RD, Simone AF, Rappaport BA (2007) Use of anesthetic agents in neonates and young children. Anesth Analg 104:509-520

6. Loepke AW, Soriano SG (2008) An assessment of the effects of general anesthetics on developing brain structure and neurocognitive function. Anesth Analg 106:1681-1707

7. Hays SR, Deshpande JK (2011) Newly postulated neurodevelopmental risks of pediatric anesthesia. Curr Neurol Neurosci Rep 11:205-210

8. Durieux M, Davis PJ (2010) The safety of key inhaled and intravenous drugs in pediatrics (SAFEKIDS): an update. Anesth Analg 110:1265-1267

9. Hansen TG, Pedersen M, Henneberg SW et al (2011) Academic performance in adolescence after inguinal hernia repair in infancy: a nationwide cohort study. Anesthesiology 114:1076-1085

10. Shu Y, Patel SM, Pac-Soo C et al (2010) Xenon pretreatment attenuates anesthetic-induced apoptosis in the developing brain in comparison with nitrous oxide and hypoxia. Anesthesiology 113:360-368

11. Nigrovic LE, Schunk JE, Foerster A et al (2011) The effect of observation on cranial computed tomography utilization for children after blunt head trauma. Pediatrics 127:1067-1073 